\title{
Crystal Lattice and Carriers Hall Mobility Relaxation Processes in Si Crystal Irradiated by Soft X-rays
}

\author{
A.J. Janavičius ${ }^{a}$, J. Storasta $^{b}$, R. Purlys ${ }^{b}$, A. Mekys $^{b}$, \\ S. BALAKAUSKAS ${ }^{c}$ AND Ž. NORGE்lA ${ }^{a}$ \\ ${ }^{a}$ Šiauliai University, Faculty of Nature \\ P. Višinskio 25, 76351 Šiauliai, Lithuania \\ ${ }^{b}$ Faculty of Physics, Vilnius University \\ Saulètekio 9, 10222 Vilnius, Lithuania \\ ${ }^{c}$ Semiconductor Physics Institute, A. Goštauto 11, 2600 Vilnius, Lithuania
}

(Received April 30, 2007)

\begin{abstract}
We applied soft X-rays for investigation of dynamics of the Frenkel point defects in a Si crystal during its saturation with metastable vacancies with neighboring $\mathrm{Si}$ atoms in excited states produced in the lattice after ejection of the Auger electrons. The irradiated irregularities and defects of the lattice cause a change of the Bragg reflection maxima. Several resonance phenomena are related to metastable states introduced into Si crystal by soft X-rays irradiation. The resonance of mean square displacements of Si atoms in the lattice and the resonance of the Hall mobility after irradiation are obtained and considered.
\end{abstract}

PACS numbers: 61.72.--y, 61.72.Dd, 05.50.+q

\section{Introduction}

Existence of relaxation processes of the Frenkel point defects and very fast diffusion of metastable lattice vacancies [1] in a Si crystal irradiated by soft X-rays were found and presented in papers [2,3]. This very important phenomenon was detected by investigation of the time dependence of the Hall mobility in a crystalline Si sample after switching off soft X-ray irradiation. The complicated time dependence of current and Hall mobility and abrupt changes of those properties after 1500 min since the moment of switch-off indicate that relaxation processes associated with metastable defects in silicon are involved. 
The main aim of our investigation is to detect relaxation processes of metastable states, using measurements of the time dependence of the diffraction maximum intensity of reflection spectrum index (333) from a crystal of orientation (111) and size $0.017 \times 0.004 \times 0.004 \mathrm{~m}^{3}$. Measurements were done with diffractometer DRON-2 (Russian device). The intensity of irradiation was registered by $\mathrm{LiF}$ dosimeter and doses of irradiation were measured by the thermoluminescent dosimeter (TLD) device [4] manufactured in the factory "Rodos" in Finland.

It is known that the Frenkel point defects and vibration of the crystal lattice influence intensity of interference lines of the diffracted X-rays and diffusion background. By measuring relative intensity of $\mathrm{X}$-ray reflection at a fixed diffraction angle $\vartheta=47.48^{\circ}$ for the $K_{\alpha 1}$ line of $\mathrm{Cu}$ X-ray characteristic spectrum (with wavelength $\lambda=1.5405 \AA$ ), we obtained dependence of the mean square displacements $\left\langle u_{\mathrm{s}}^{2}\right\rangle$ of Si atoms from their regular equilibrium positions in crystal lattice on time. The resonance-like behavior of the time dependence of $\left\langle u_{\mathrm{s}}^{2}\right\rangle$ can be explained by the presence of excited atoms in the metastable states [1] in a Si crystal irradiated by soft X-rays. Also we measured that the resonance-like behavior of the generated point defects creates some resonant phenomenon in electrical conductivity and the Hall mobility.

\section{Measurements of dynamics of the Frenkel point defects in Si crystal irradiated by soft X-rays}

Influence of X-ray generated vacancies on peaks of a diffraction spectrum is twofold. When the vacancies penetrate into the bulk of the sample, its crystalline structure may change. The increase in vacancy concentration can be accompanied by a decrease in the amount of material corresponding to the initial crystalline phase. As a result, diffraction spectrum peak intensity of the Bragg scattering also decreases. Quantitative analysis of crystalline structure is based on the investigation of intensity [5] $I_{h k l}$ of X-rays reflected from the crystal lattice, with reflection indexes $h k l$ :

$$
I_{h k l}=I_{0} F_{h k l}^{2} L P A(\vartheta, \mu) p V
$$

where $I_{0}$ is the intensity of incident beam, $\mu$ is the coefficient of absorption of $\mathrm{X}$-rays, $F_{h k l}^{2}$ is the structure factor, $L P$ is the product of Lorentz and polarization factors, $A$ is the absorption factor, $p$ is the multiplicity factor, $V$ is the scattering volume. In result, the thermal motion of atoms, defects of the lattice can be evaluated by decreasing the relative intensity of diffraction maximum

$$
I_{i} / I_{0}=\exp \left(-\frac{16}{3} \pi^{2}\left\langle u_{\mathrm{i}}^{2}\right\rangle(\sin \vartheta / \lambda)^{2}\right),
$$

where $\left\langle u_{\mathrm{i}}^{2}\right\rangle$ is an initial mean square deviation of atoms from regular pattern. The displacements of atoms to metastable or interstitial positions produce vacancies in the crystal lattice caused by the Auger effect [6]. These displacements are unrelated processes and depend only on time and parameters of X-rays. In this case we can represent the total mean square deviation

$$
\left\langle u(t)^{2}\right\rangle=\left\langle u_{\mathrm{i}}^{2}\right\rangle+\left\langle u(t)_{\mathrm{V}}^{2}\right\rangle
$$


like a sum of $\left\langle u_{\mathrm{i}}^{2}\right\rangle$ and mean square deviation $\left\langle u(t)_{\mathrm{V}}^{2}\right\rangle$ of vacancies and displaced atoms, which depends on irradiation time. Substituting (2.3) instead of $\left\langle u_{\mathrm{i}}^{2}\right\rangle$ into (2.2) we obtain

$$
I(t) / I_{0}=\exp \left(-\frac{16}{3} \pi^{2}\left(\left\langle u_{\mathrm{i}}^{2}\right\rangle+\left\langle u(t)_{\mathrm{V}}^{2}\right\rangle\right)(\sin \vartheta / \lambda)^{2}\right) .
$$

Dividing (2.4) by (2.2) we can obtain the square root of mean square deviation of atoms dependence on time in the crystal lattice

$$
\sqrt{\left\langle u(t)_{\mathrm{V}}^{2}\right\rangle}=\sqrt{\ln \left(I_{\mathrm{i}} / I(t)\right)} /\left(\frac{4 \pi}{\sqrt{3}} \frac{\sin \vartheta}{\lambda}\right)
$$

by influence of point defects generated by soft X-rays.

In the case of the Auger effect, kinetic energy of the ejected electron, called an Auger electron, is equal to the energy of the corresponding X-ray photon minus the electron binding energy. The highest probability of the Auger effect and the largest amount of point defects produced by this effect in Si crystal can be received in the case of X-ray photons with $6 \mathrm{keV}$ energy [6]. The Frenkel point defects are received as a result of the Coulomb-force interactions and transitions of the ionized $\mathrm{Si}$ atoms from their equilibrium sites to metastable or interstitial states. The relative fluctuations of the number of atoms in excited states can be related to relative fluctuations of crystal volume containing $N_{\text {mi }}$ atoms

$$
\Delta V(t) \approx N_{\mathrm{mi}}(t) a^{3} .
$$

Here, $a=5.431 \AA$ is an Si lattice parameter in vacuum at $295.6 \mathrm{~K}$.

Taking into account the fact that fluctuations of the crystal volume can be related to changes of $\left\langle u(t)_{\mathrm{V}}^{2}\right\rangle$ we obtain that changes of volume

$$
\Delta V(t) \approx N\left(\sqrt{\left\langle u(t)_{\mathrm{V}}^{2}\right\rangle(t)}\right)^{3}
$$

depend on time. Here $N=5 \times 10^{22} \mathrm{~cm}^{-3}$ is the number of $\mathrm{Si}$ atoms in the unit volume. From formulae (2.6) and (2.7) we obtain

$$
N_{\mathrm{mi}}(t) \approx N\left(\sqrt{\left\langle u(t)_{\mathrm{V}}^{2}\right\rangle(t)}\right)^{3} / a^{3} .
$$

After 6 hours duration of irradiation of the sample, we detected a decrease in maximum intensity without changing half of width of diffraction peak. This indicates that the X-rays generate only point defects [5]. After stoppage of irradiation in 50-70 hours, the crystal lattice of $\mathrm{Si}$ was restored. This fact shows that metastable vacancies and excited point defects in Si crystals can be generated not only by action of $1-2 \mathrm{MeV}$ electrons [7] or ${ }^{60} \mathrm{Co}$ gamma rays [8] but also with soft X-rays $[2,3]$. The recovery of Si crystal after irradiation is not complete and this fact is related to interstitial atoms or some stable complexes, whose amount increases during irradiation. Consequently, we can express the mean square deviation of lattice from the regular pattern, resulting from atom transitions into metastable or interstitial states, in the following way:

$$
\left\langle u(t)_{\mathrm{V}}^{2}\right\rangle=\left\langle u_{\mathrm{dv}}^{2}\right\rangle+\left\langle u_{\mathrm{di}}^{2}\right\rangle .
$$


The mean square deviation $\left\langle u_{\mathrm{dv}}^{2}\right\rangle$ depends on the number of excited vacancies and atoms in metastable states and on the time. $\left\langle u_{\mathrm{di}}^{2}\right\rangle$ depends on the number of interstitial atoms. The number of interstitial atoms is proportional to irradiation dose.

The vacancy formation energy can be calculated as follows [9]:

$$
\Delta E=E_{\mathrm{v}}-\frac{n-1}{n} E_{\mathrm{b}},
$$

where $E_{\mathrm{v}}$ is the total energy of the cell containing the vacancy, $n$ is the number of atoms in the cell, and $E_{\mathrm{b}}$ denotes the total energy of the bulk calculated using the same cell parameters. When after the irradiation the crystal lattice has a non-equilibrium number of interstitial atoms and associated vacancies, the crystal is in the excited state and energy of the crystal is increased by $n_{\mathrm{vi}} \Delta E_{\mathrm{i}}$. Here $n_{\mathrm{vi}}$ and $\Delta E_{\mathrm{i}}$ are the number and the energy of the produced pairs of interstitial atoms and excited vacancies in the process of irradiation. In result of energy release in the carriers capture process by vacancies and vacancies recombination with the interstitial atoms we have energy conversion to the vibration modes of $\mathrm{Si}$ lattice. Also the crystal energy increases by $n_{\mathrm{ve}} \Delta E_{\mathrm{e}}$ by the Auger effect in result of generation of $n_{\mathrm{ve}}$ excited vacancies with neighboring atom in the excited state. The total energy $\Delta E_{\mathrm{f}}$ absorbed in the crystal can be approximately expressed as the sum of the two terms involving generation energies of the Frenkel point defects and the term reflecting energy loss in the process of crystal lattice transition into the thermal equilibrium state:

$$
\Delta E_{\mathrm{f}}(t)=n(t)_{\mathrm{vi}} \Delta E_{\mathrm{i}}+n(t)_{\mathrm{ve}} \Delta E_{\mathrm{e}}+\Delta E_{\mathrm{m}}(t) .
$$

The term $\Delta E_{\mathrm{m}}(t)$ represents the time dependent energy loss related to transitions of metastable states of the Frenkel point defects into stable states. The numbers of atoms in metastable and interstitial states depend on time in a different manner, and we can measure a relaxation process of the relative intensity $I / I(t)$ of diffraction maximum caused by time dependence of the number of the Frenkel point defects composed of the vacancies and neighboring atoms in metastable or interstitial states.

\section{Compound relaxation processes in excited systems}

For investigation of the temperature dependence of kinetic parameters (time, frequency, concentration, parameters of diffusion, and Brownian motion) of compound systems, the Arrhenius law is usually applied [4]:

$$
\Phi_{n}(T)=A \exp \left( \pm U_{n} / k T\right),
$$

where $\Phi_{n}(T)$ - diffusion coefficient, $T$ - temperature $A$ - pre-exponential factor.

The activation energy $U_{n}$ terms can be determined from the experimental measurements of $\ln \Phi_{n}(1 / T)$. The usually obtained values of $U_{n}$ are $5-10 \mathrm{eV}$. Those values are larger than the true interaction energy between atoms or molecules in condensed systems, where this term is usually equal to about $1 \mathrm{eV}$. This can be explained only when $U_{n}$ is not a potential barrier for elementary event, but 
a sum of potential barriers of elementary acts of compound collective processes instead [4].

In this case, the probability of producing vacancies by compound processes caused by irradiation of Si crystal with soft X-rays can be represented by the following formula [4]:

$$
W=\sum_{n} W_{n} \exp \left(-E_{n} /(k T)\right) .
$$

Here, $W_{n}$ is a probability of transition from the crystal site with energy $E_{n}$ to the interstitial or metastable state [1]. This formula can also describe the reverse process. In the case of a two-level system, we can use the Boltzmann statistics for calculation of population ratio $P[10]$ of two quantum levels with energies $\varepsilon_{2}>\varepsilon_{1}$ :

$$
P=\omega\left(\varepsilon_{2}\right) / \omega\left(\varepsilon_{1}\right)=\exp \left(-E_{21} /(k T)\right),
$$

where $E_{21}=\varepsilon_{2}-\varepsilon_{1}, E_{21}>0$. For an excited system, $P \geq 1$, and then we have $T<0$.

It is possible to describe properties of an excited system in the non-isothermal case by introducing the following temperature function [10]:

$$
T(t)=\frac{1}{a-b \ln (t c)} .
$$

The temperature function $T(t)$ depends on parameters $a, b, c$ and has a singularity at the time moment $t_{0}$. We can express time $t$ in the units of time moment $t_{0}$, then we obtain

$$
t=z t_{0}, \quad T(t)=-1 /(b \ln (z)), \quad t_{0}=\left(\mathrm{e}^{a / b}\right) / c .
$$

The temperature function $T(t)$ corresponds to the Boltzmann statistic for description of the population ratio $P(t)(3.3)$ of two quantum levels $E_{2}>E_{1}$ :

$$
P(t)=z^{E_{21} b / k}=z^{\alpha-1}, \quad \alpha=1+(E b) / k .
$$

$E$ is the activation energy of the diffusion in solids.

From the last expression, if $b<0$ and $\alpha<1$, we obtain that all excited vibration states $E_{2}$ of atoms taking part in diffusion are populated at the initial time when $z \rightarrow 0$. When $b>0$ and $\alpha>1$, we have the process of saturation of the system by the excited states. The parameter $b$ depends on intensity and spectrum of X-rays used for irradiation of the crystal. Vacancies are produced by moving the atoms from the lattice site to metastable state [4] with $\varepsilon_{2}=1 \mathrm{eV}$ energy or to the interstitial state as a result of the Coulomb interaction after the ejection of the Auger electrons. For displacement of these atoms to metastable states, they must penetrate through the barrier of height $\Delta E=1.5 \mathrm{eV}$ [5], and for displacement to interstitial state [2] with energy $0.8 \mathrm{eV}$, they must penetrate through the additional potential barrier of height $0.5 \mathrm{eV}[5]$.

Then, using (3.3) and (3.6), we can obtain

$$
\frac{N_{\mathrm{m}}(z)}{N}=\frac{z^{\alpha-1}}{z^{\alpha-1}+1} \text {. }
$$


Here, $N_{\mathrm{m}}$ is the number of $\mathrm{Si}$ atoms in metastable and interstitial states, $N$ is the number of $\mathrm{Si}$ atoms in $\mathrm{cm}^{3}$.

If we have periodic excitation of metastable system with relaxation period $\tau_{\mathrm{i}}=t_{\mathrm{f}}-t_{\mathrm{i}}$, we can present (3.7) in the following way:

$$
\frac{N_{\mathrm{m}}(t)}{N}=\frac{\left(\frac{t-t_{\mathrm{i}}}{t_{0}}\right)^{\alpha-1}}{\left(\frac{t-t_{\mathrm{i}}}{t_{0}}\right)^{\alpha-1}+1}, \quad t_{\mathrm{i}} \leq t \leq t_{\mathrm{f}} .
$$

\section{Relaxation processes initiated by soft X-rays in crystalline silicon}

In our experiments we used the crystal sample of high resistance $667 \Omega \mathrm{cm}$ Si doped with boron. If the Si crystal is doped with boron the three electrons form covalent bond with neighboring Si atoms, leaving the fourth bond broken. The irradiation of the Si crystal with soft X-rays initiates the Auger processes which generates the very fast vacancies $[2,3]$ and breaks bonds in the lattice. All broken bonds change the energy of lattice and increase lattice distortions. Carrier density dependence on the Auger recombination, carrier's transitions to charged vacancies and phonon-assisted Auger recombination [11] makes expedient to investigate the influence of these processes dependence of lattice parameters on irradiation. Fast non-radiative Auger recombination and free carriers absorption prevent optical transitions in crystal silicon [12] and increase probability of relaxation effects in lattice. These relaxation effects also can be generated by the different diffusivity of vacancies in different charge states. For diffusion of charged vacancies like in diffusion experiments [13] with the Si samples doped by $\mathrm{B}$ and $\mathrm{P}$ the Fermi level $E_{\mathrm{F}}$ must depend on time. It is important that concentration of the charged defects also varies with $E_{\mathrm{F}}[13]$.

Experimental measurements of intensity of X-ray reflection were performed with diffractometer DRON-2.0 by summing the number of photons in $10 \mathrm{~s}$ time interval with $1 \mathrm{~min}$ period for a fixed diffraction angle $\vartheta=47.48^{\circ}$. The experiment was done using a $\mathrm{Cu}$ anode with X-ray characteristic spectral line $K_{\alpha 1}$ of wavelength $\lambda=1.5405 \AA$ for anode voltage $6 \mathrm{kV}$ and Roentgen tube current $23 \mathrm{~mA}$, respectively. The measurements were performed at the temperature of $290 \mathrm{~K}$. Results are presented in Fig. 1. We calculated and presented in Fig. 2 the square root $i=\sqrt{\left\langle u_{\mathrm{d}}\right\rangle^{2}}$ of the mean square displacements (2.5) of Si atoms from their regular equilibrium positions in crystal lattice dependence on time of irradiation. The oscillating terms $i$ of these displacements were fitted with the formula (3.8) for the time dependence of saturation of Si crystal lattice with metastable states, using a fixed value of the excitation parameter $\alpha=0.8$. These oscillations with the same excitation parameter $\alpha$ show that Si crystal lattice irradiated by soft X-rays acts as a two-level system [10] for atomic transitions from metastable states into neighboring metastable vacancies or the reverse transitions by producing metastable vacancies. Also there can be produced the simple vacancies 


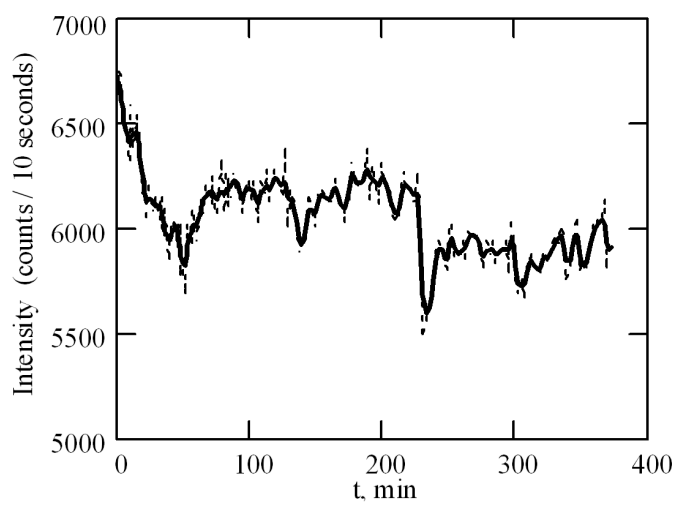

Fig. 1. Experimental dependence of intensity (number of photons registered in $10 \mathrm{sec}-$ onds with diffractometer DRON-2.0 for a fixed diffraction angle $\vartheta=47.48^{\circ}$ ) of reflection from (111) oriented Si crystal on time $t$ (min). Experiment was done with $\mathrm{Cu}$ anode X-ray characteristic spectral line $K_{\alpha 1}$ of wavelength $\lambda=1.5405 \AA$ for anode voltage $6 \mathrm{kV}$ and X-rays tube current $23 \mathrm{~mA}$.

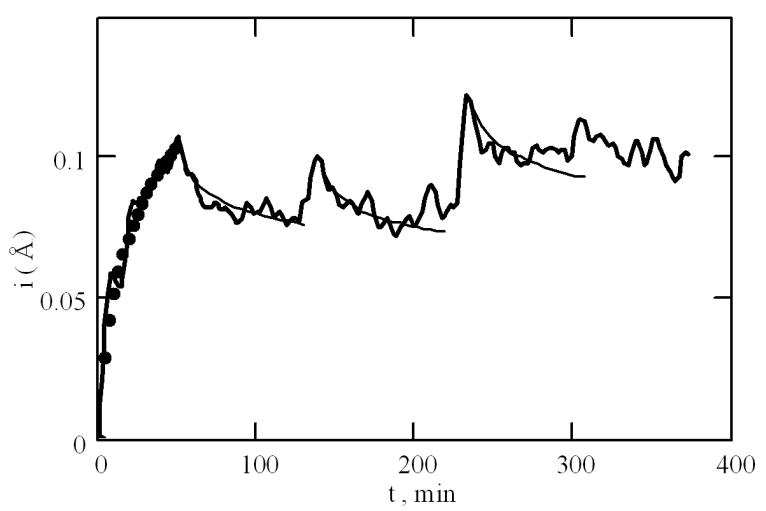

Fig. 2. Dependence of square root of mean square displacements $i=\sqrt{\left\langle u_{\mathrm{d}}^{2}\right\rangle}$ of atoms from equilibrium positions on time $t$ (min). Thin lines are interpolations of $i$ and describe periodic transitions $(\alpha=0.8)$ from metastable states (3.8) with equal time periods $t_{0}=80 \mathrm{~min}$ into lower metastable state of $\mathrm{Si}$ crystal. Interpolation line from dots is proportional to the excitation function (3.8) with excitation parameter $\alpha=1.65$ and has period $t_{0}=50 \mathrm{~min}$. X-rays tube current $23 \mathrm{~mA}$ anode voltage was $6 \mathrm{kV}$.

with neighboring atoms in the interstitial sites. All these processes are generated by electron transitions between conductivity band and charged vacancies and excited atoms vibrations in the metastable states. Importance of excited lattice vibrations can be derived from the fact that Si crystal after irradiation can stay in excited states for many hours $[2,3]$. The initial part of dependence of the point defect-producing process on irradiation time for our experiment can be defined by 
the excitation parameter $\alpha=1.65$. The equal constants of excitation for different anode voltages and the Roentgen tube currents demonstrate that these constants depend only on properties of $\mathrm{Si}$ crystal. The obtained resonance of the term $i$ can be interpreted as normal modes of resonant transitions of coupled oscillating atoms into the metastables states and the reverse transitions into vacancies in the process of the irradiation by $5-15 \mathrm{keV}$ photons. These photons can increase the vibration energy of the lattice and produce excited vacancies by means of the Auger effect. The vibrations of atoms in excited states of lattice generated by $\mathrm{X}$-rays play the role of external periodic force, and when its frequency coincides with the frequency of thermal vibrations of atoms placed at neighboring of excited vacancies [14] $\omega_{\mathrm{v}}=\sqrt{3 k / m-\gamma^{2}}$, the resonance occurs. Here, $k$ is oscillators constant, $\gamma=c / 2 m$ where $c$ is the coefficient of velocity-dependent damping force, $m$ is the oscillators mass. The maximum amplitude of oscillations of vacancies of neighboring atoms occurs at the resonant frequency $\omega_{\mathrm{r}}=\omega_{\mathrm{v}}$. This resonance can be obtained only for special values of anode current. The probability of the Auger effect and vacancy formation depends on the anode voltage. It follows that oscillations of the number of point defects in Si lattice can be measured only for special values of voltage and current in a Roentgen tube.

\section{The oscillations of Hall mobility}

The carriers colliding with phonons generated from lattice oscillations are exchanging with them by energies and quasi-impulses. Local deformations in the lattice produced by recombination of generated vacancies and carriers transitions can produce waves, which must change the energies of valence and conductivity bands [15]. Vacancies are rapid diffusers [16], with activation energies of migration: $0.18 \mathrm{eV}$ for $\mathrm{V}^{2-}, 0.45 \mathrm{eV}$ for $\mathrm{V}^{0}, 0.32 \mathrm{eV}$ for $\mathrm{V}^{2+}$. Usually it is accepted that energy levels of $\mathrm{V}^{-}$and $\mathrm{V}^{+}$have intermediate meanings. During diffusion vacancies and self-interstitials may recombine with the vacancies and interact with the impurities. Also vacancies can trap carriers changing its charge, energies and diffuse out of the samples. We measured [3] that negative vacancies generated in Si crystal by soft X-rays can leave the sample $3.3 \times 3.1 \times 3.1 \mathrm{~mm}^{3}$ during the time $t=87 \mathrm{~min}$. For the Hall mobility measurements we used the samples of the thickness $0.3 \mathrm{~mm}$. We performed the measurements at the temperature of $290 \mathrm{~K}$. The measured time dependence of the Hall mobility presented in Fig. 3 for $p$-type Si samples can be explained using energy levels of vacancies presented in paper [7]. Although the vacancies in these experiments were generated with electrons irradiation at $4.2 \mathrm{~K}$ and disappear at temperatures lower than $200 \mathrm{~K}$ we can use the obtained results at room temperature, because energy transmission to crystal lattice by the Auger effect is significantly smaller. Capture of the electrons from conductivity band and providing recombination with the holes the vacancies $\mathrm{V}^{+}$, $\mathrm{V}^{2+}$ transmit energy to the lattice generating athermal vibrations of the nearest atoms. Vacancies $\mathrm{V}^{0}$ generated by X-rays at the surface of the sample with activation energies of migration $0.45 \mathrm{eV}$ are slowly spreading to the volume decreasing 


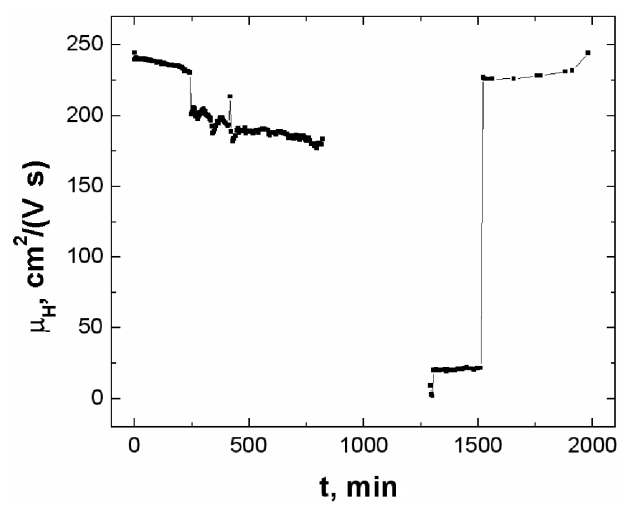

Fig. 3. Hall mobility relaxation in $p$-type Si crystal boron doped sample (Hall mobility $\left.\mu_{0 \mathrm{H}}=240 \mathrm{~cm}^{2} /(\mathrm{V} \mathrm{s}), p_{0}=4 \times 10^{13} \mathrm{~cm}^{-3}, \sigma_{0}=1.51 \Omega^{-1} \mathrm{~cm}^{-1}\right)$ irradiated by $20 \mathrm{kV}$, $20 \mathrm{~mA}$ X-rays in $3.6 \mathrm{~h}$ time from the side $1.5 \times 4.5 \mathrm{~mm}^{2}$. Sample width $0.4 \mathrm{~mm}$, distance between current contacts $4.65 \mathrm{~mm}$, width between Hall contacts $1.55 \mathrm{~mm}$. Voltage between the current leads is $19.4 \mathrm{~V}$ and the magnetic field induction $B=1.7 \mathrm{~T}$.

the Hall mobility. We measured very slowly decreasing Hall mobility for $p$-type Si crystal samples and presented results in Fig. 3. According to measurements provided in [7] vacancies $\mathrm{V}^{+}, \mathrm{V}^{2+}$ interacting with lattice relaxation periodically change their positions in the bands gap. We can see in Fig. 2 that after $3 \mathrm{~h}$ irradiation the large lattice relaxation was achieved. Also we watch in Fig. 3 the similar abruptly changes with the same period of the Hall mobility. We can suppose that these changes are a result of the abruptly changes of energies and distribution in the bands gap of charged vacancies [7]. There was shown that positions of the energy levels of vacancies $\mathrm{V}^{+}, \mathrm{V}^{2+}$ in the bands gap can be interchanged by lattice relaxation. The frequent oscillations Hall mobility and conductivity with periods $50 \mathrm{~ms}$ or longer singly oscillations with $150 \mathrm{~ms}$ relaxation periods were measured in boron doped sample [2] and Czochralski grown $n$-type Si crystal sample. These relaxations with short period can be connected with some resonance of multiphonon transitions in lattice [15] generated from electron transitions between carriers and vacancies [7]. The measurements were provided with Russian device "B7-30". These results are presented in Figs. 3 and 4. It is interesting to remark that after $1500 \mathrm{~min}$ electrical properties recover. According to the paper [17] we can suppose that in $p$ - and $n$-samples mostly negative and positive vacancies can be produced by the Auger effect as a result of the Coulomb interaction with the ions of impurities. Also we can suppose that neutral vacancies can be produced by the Auger effect and Raman scattering of neighbor silicon atoms [18]. As a result of the carriers transitions, finally we have the same vacancies [7] like the obtained by electron irradiation. The decreasing Hall mobility for $p$-type samples in Fig. 3 shows that lifetimes of carriers are defined by scattering on most slowly diffusing [19] neutral vacancies. From measurements presented in Fig. 3 we can deduce 


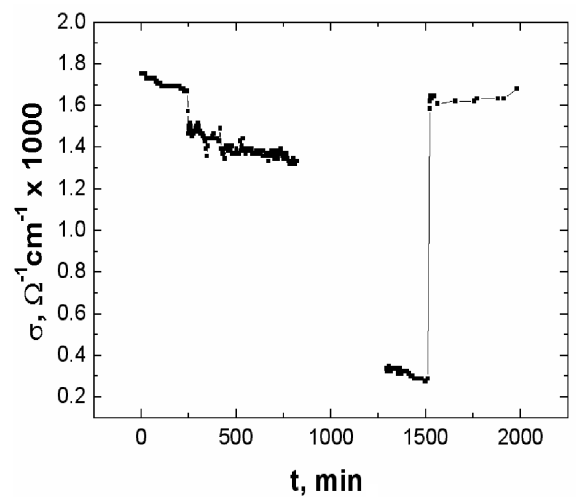

Fig. 4. Relaxation of conductivity in $p$-type Si crystal boron doped sample (Hall mobility $\left.\mu_{0 \mathrm{H}}=240 \mathrm{~cm}^{2} /(\mathrm{V} \mathrm{s}), p_{0}=4 \times 10^{13} \mathrm{~cm}^{-3}, \sigma_{0}=1.51 \Omega^{-1} \mathrm{~cm}^{-1}\right)$ irradiated by $9 \mathrm{kV}$, $20 \mathrm{~mA}$ X-rays in $3.6 \mathrm{~h}$ time from the side $1.5 \times 4.5 \mathrm{~mm}^{2}$. Sample width $0.4 \mathrm{~mm}$, distance between current contacts $4.65 \mathrm{~mm}$, width between Hall contacts $1.55 \mathrm{~mm}$. Voltage between the current leads is $19.4 \mathrm{~V}$ and the magnetic field induction $B=1.7 \mathrm{~T}$.

that after 1500 min we have Hall conductivity recovery induced by multiphonon transition between positive vacancies and electrons of conductivity band.

In Fig. 4 we see that the recovery of electric properties after $1500 \mathrm{~min}$ is not full and we can suppose that not all neutral vacancies left the sample. Comparing Figs. 3 and 4 we see that conductivity $\sigma=e n \mu$ and mobility $\mu$ are changing in the same way. Taking into account [15] that mobility can be obtained dividing the Hall mobility on constant $r=1.18 \div 1.93$, which depends on carriers scattering way, we can confirm that conductivity and Hall mobility for $p$-type [2] Si samples depend on the same processes associated with metastable vacancies in the crystal silicon.

\section{Discussion and conclusions}

Using soft X-rays under conditions of the Auger effect [5], we obtained periodic transitions of Si atoms into lower metastable states with smaller distortions of the lattice and the reverse transitions. Those transitions occur due to influence of lattice vibrations on neighboring vacancies. In recent papers $[2,3]$ it was shown that after removing $\mathrm{Si}$ atoms from the sites in the Si lattice into metastable or more stable interstitial states, negatively charged vacancies are formed, which can move long distances relatively fast. Those vacancies can migrate if at least two more bonds are broken [15], for which an energy of $0.35 \mathrm{eV}$ is needed at room temperature. The energy emission after transition of an Si atom from metastable state into the vacancy is about $1 \mathrm{eV}[5]$ and is more than sufficient for the motion of vacancies. Some atoms, however, in the metastables states, which are neighboring to excited vacancies, can transit into more stable interstitial states changing these vacancies into unexcited. The prevailing direction of these processes depends 
on interaction with the lattice vibrations and electrons transitions. The periodic resonant distortions of the Si lattice presented in Fig. 2 were obtained in the process of irradiation of the crystal with soft X-rays for copper anode with voltage $U=6 \mathrm{kV}$ and current $23 \mathrm{~mA}$. This periodic process of population of metastable states and generation of excited vacancies can be explained in terms of periodic excitations of a two-level system [10] formed by atoms in metastable states of $\mathrm{Si}$ lattice, produced by the Auger effect and lattice vibrations interference. From electron binding energy [16] $E=1844 \mathrm{eV}$ in $K$ shell of Si atom [13] and maximum intensity of continuous spectrum of X-rays [5] for wavelength $\lambda=18.517 / U$, we can evaluate the minimum anode voltage $U_{\min }=2.754 \mathrm{kV}$ required for occurrence of the Auger effect.

However, in order to obtain the periodic resonant distortions of Si lattice, a certain amount of energy should be also transmitted into the lattice for generation of periodic resonant atom transitions from metastable states into vacancies and activation of vacancy motion. For realization of this aim, appropriate currents in the Roentgen tube must be used.

Results presented in Fig. 2 indicate that square root of the mean square displacement $i$ is significantly oscillating: $i(138 \mathrm{~min})=0.1 \times 10^{-10} \mathrm{~m}, i(218 \mathrm{~min})=$ $0.075 \times 10^{-10} \mathrm{~m}$. Using formula $(2.8)$ we can calculate the oscillations of concentration of $\mathrm{Si}$ atoms in metastable states for the following time intervals: $t=130-218 \mathrm{~min} ; t=226-310 \mathrm{~min}$ :

$$
\begin{aligned}
& N_{\mathrm{mi}}(130 \mathrm{~min})=1.316 \times 10^{17} \mathrm{~cm}^{-3}, \quad N_{\mathrm{mi}}(138 \mathrm{~min})=3.121 \times 10^{17} \mathrm{~cm}^{-3}, \\
& N_{\mathrm{mi}}(130 \mathrm{~min})=N_{\mathrm{mi}}(218 \mathrm{~min}), \quad \Delta E_{\mathrm{mi}}\left(t_{2}, t_{1}\right)=\left[N_{\mathrm{mi}}\left(t_{2}\right)-N_{\mathrm{mi}}\left(t_{1}\right)\right] \Delta E_{\mathrm{a}} \\
& N_{\mathrm{mi}}(226 \mathrm{~min})=1.598 \times 10^{17} \mathrm{~cm}^{-3}, \quad N_{\mathrm{mi}}(233 \mathrm{~min})=5.529 \times 10^{17} \mathrm{~cm}^{-3} \\
& N_{\mathrm{mi}}(244 \mathrm{~min})=3.121 \times 10^{17} \mathrm{~cm}^{-3}, \quad N_{\mathrm{mi}}(310 \mathrm{~min})=2.6709 \times 10^{17} \mathrm{~cm}^{-3} .
\end{aligned}
$$

In the time interval $t=226-310$ min we can see point defects transitions like some resonance of lattice. We can express the fast absorption of energy by increasing number of point defects $\Delta E_{\mathrm{mi}}\left(t_{2}, t_{1}\right)=\left[N_{\mathrm{mi}}\left(t_{2}\right)-N_{\mathrm{mi}}\left(t_{1}\right)\right] \Delta E_{\mathrm{a}}$.

Transitions of Si atoms from lattice sites into metastable states and the reverse transitions involve absorption or emission of the energy amount $\Delta E_{\alpha}=1 \mathrm{eV}$ per one Si atom [5]. Thus, for transitions from metastable states into vacancies or the reverse transitions we can calculate the difference of absorbed and lost energies $\Delta E_{\mathrm{mi}}\left(t_{2}, t_{1}\right)=\left[N_{\mathrm{mi}}\left(t_{2}\right)-N\left(t_{1}\right)\right] \Delta E_{\alpha}$. Now we can evaluate the emitted energies for oscillations presented in Fig. 2: $\Delta E_{\mathrm{mi}}(138 \mathrm{~min}, 218 \mathrm{~min})=2.892 \times 10^{4} \mathrm{~J} / \mathrm{m}^{3}$. Considering second time interval more carefully we obtained two different resonances of lattice. We measured the saturation of Si sample $0.3 \times 0.3 \times 1.7 \mathrm{~cm}^{3}$ through the side $0.3 \times 1.7 \mathrm{~cm}^{2}$ with irradiated vacancies. This corresponds to the increase in the mean square displacements of atoms in Fig. 2 at $t=50$ min. Irradiation was done through the open aperture with area $0.05 \times 1.5 \mathrm{~cm}^{2}$; the irradiation dose was $D=1.9668 \times 10^{5} \mathrm{mSv} /\left(\mathrm{s} \mathrm{mA} \mathrm{m}^{2}\right)$, measured with LiF thermoluminescent dosimeter [20]. After irradiation time $t=50 \mathrm{~min}$ it was found that 
the square root of the mean square displacement was $i=0.11 \times 10^{-10} \mathrm{~m}$, and from (2.8) we obtain $N_{\mathrm{mi}}(50 \mathrm{~min})=4.154 \times 10^{17} \mathrm{~cm}^{-3}$. Taking into account volume of a sample and irradiation dose $D$, we calculated the energy absorbed by the sample: $\Delta E=4.731 \times 10^{-2} \mathrm{~J}$. We obtained numbers of produced vacancies in the two time intervals: (130-138 $\mathrm{min})$; (226-233 $\mathrm{min})$ are $2.759 \times 10^{16}$ and $6.015 \times 10^{16}$, respectively. The energy used for producing one vacancy with soft X-rays in the sample is $4.507 \mathrm{eV}$. Also in this case we can evaluate the density $3.845 \times 10^{17} \mathrm{~cm}^{-3}$ of produced vacancies. The realistic vacancy producing energy is about $1 \mathrm{eV}[5]$ and in our case we see that most of energy absorption is related to lattice excitations of Si crystal. We think that relaxation processes in Si crystals excited by soft X-rays is one of main reasons of very fast diffusion, or superdiffusivity [10], of vacancies, which has been measured in [17], as well as the electron-hole recombination in $\mathrm{Si}$ crystals [18]. However, in the latter work, like in later works [21-23], vacancies were obtained by irradiation with 1-3 MeV electrons, gamma rays, or fast neutrons. In our case, we obtained superdiffusion [10] of metastable excited vacancies generated with soft X-rays [2,3] as a consequence of the Auger effect [24]. The diffusion coefficients for these excited vacancies $[17,22]$ are $10^{4}$ times greater than for the vacancies obtained by the thermal excitation. The energies of the Auger electrons for $\mathrm{Si}$ are less than $1.8 \mathrm{keV}$, and those electrons can lose energy by ionization, excitation, and multiphonon nonradiative transitions [22] of atoms which are surrounding the produced vacancies. These nonradiative transitions are the main reason of large intensity fluctuations presented in Fig. 1. We assume that fluctuations cannot be directly connected with increasing density of point defects.

We obtained very fast excited vacancies with long lifetimes [3]. In the paper [7] it was established that a vacancy has five different charge states $\mathrm{V}^{2+}, \mathrm{V}^{+}$, $\mathrm{V}^{0}, \mathrm{~V}^{-}, \mathrm{V}^{2-}$ and increasing energies in the silicon band gap. The intensities relaxations presented in Fig. 1 is a result of lattice oscillations [7] generated by broken bond reconstruction in different ways. Levels for $\mathrm{V}^{2+}, \mathrm{V}^{+}$are $0.03 \mathrm{eV}$ and $0.13 \mathrm{eV}$ above the valence band edge. Taking into account that minimum indirect energy gap at $300 \mathrm{~K}$ for $\mathrm{Si}$ crystal is $1.12 \mathrm{eV}$ we can evaluate that the energies of electron transitions from conductivity band to these vacancies are sufficient for generation of very fast negatively charged vacancies and large lattice relaxation which we found experimentally. Also these periodic processes depend on different velocities of diffusion of generated vacancies in different charge states, dependence of the Fermi level and density of charge carriers on time. According to Van Vechten [22] the initial part of dependence of values of vacancy migration from temperature can be explained introducing short-range forces breaking the covalent bonds and longrange forces. We can suppose that in our case we have the similar situation. The very fast oscillations of intensity of reflected photons presented in Fig. 1 depend on short-range forces generated by the Auger effect. Slow oscillations of middle mean square displacements of atoms in the Si lattice presented in Fig. 2 depend on long-range Coulomb forces acting between diffusing charged vacancies in the 
lattice. Silicon, like many semiconductor materials, has small amplitudes of atoms vibration [5] because of the strong $s p^{3}$ hybridized bonding within an extended lattice. This fact and high Debye temperature are reasons of weak dependence of the root of mean square displacement of the atoms on temperature.

The main conclusion following from results presented in $[11,21,25]$ is the relation between superdiffusivity of metastable vacancies and lattice relaxation of $\mathrm{Si}$ crystals excited by soft X-rays. We obtained that after $3 \mathrm{~h}$ irradiation the large relaxation of lattice was achieved. After irradiation we measured abruptly changes of the Hall mobility for $p$-type samples with the same $3 \mathrm{~h}$ period and recovery of mobility after 1500 min. We can suppose that this can be governed by charging and recharging processes of vacancies and tied lattice relaxation [26].

It is known that Frenkel pairs produced by electrons, irradiation of $\mathrm{MeV}$ energies [27] and $\gamma$ irradiation have a very high mobility at low temperatures. An opposite situation was obtained by investigations of the self diffusion in the high temperatures region [28].

For the obtaining of mobile Frenkel defects of high concentration at room temperature we must create defects with low energies irradiation [28]. Providing experimental investigations were shown, the Frenkel defects play more important role than divacancies, clusters and complexes [28]. We suppose that, in our case, when excitation energies are small, the chemical bonds of $\mathrm{Si}$ atoms, which are moved from equilibrium sites, are not broken. When Si crystals are excited by $\mathrm{X}$-rays the electrical conductivity is changed only by vacancies. Producing the Frenkel defects, according to [3], is sufficient to transmit 10-30 eV energy to the site atom, which coincides with results obtained in paper [29]. We obtained the Frenkel defects as a result of the Auger electron transitions and lattice vibrations. Those processes can occur like the result of carriers transitions into deep energy levels of defects [29]. The Frenkel defects can be easily generated when the Auger transitions occur in atoms whose neighbor is charged impurity atom. For the Frenkel defects produced by the soft X-rays we got the negative vacancies and did not register the charged interstitials in our measurements of conductivity $[2,3,21,25]$. The concentrations of obtained negative vacancies are slightly less than that of boron atoms in Si samples [2].

\section{References}

[1] B.N. Mukashev, X.A. Abdulin, Y.V. Gorelkinski, Adv. Phys. Sci. 170, 143 (2000) (in Russian).

[2] R. Purlys, A.J. Janavičius, A. Mekys, S. Balakauskas, J. Storasta, Lith. J. Phys. 41, 376 (2001).

[3] A.J. Janavičius, J. Banys, R. Purlys, S. Balakauskas, Lith. J. Phys. 42, 337 (2002).

[4] A.I. Slucker, Y.I. Polikarpov, K.V. Vasiljeva, Phys. Solid State 44, 1529 (2002).

[5] A.F. Chochlov, Physics of Solid State, Vol. 1, High School, Moscow 2001, p. 365 (in Russian). 
[6] V.S. Vavilov, A.E. Kiv, O.R. Nijazova, Mechanism of Producing and Migration of Defects in Semiconductors, Science, Moscow 1981, p. 368.

[7] G.D. Watkins, Mater. Sci. Semicond. Proc. 3, 227 (2000).

[8] L.F. Makarenko, Physica B: Condens. Matter 308, 465 (2001).

[9] T.R. Mattson, A.E. Mattson, Phys. Rev. B 66, 214110 (2002).

[10] A.J. Janavičius, Acta Phys. Pol. A 93, 505 (1998).

[11] W. Bardyszewski, D. Yevick, J. Appl. Phys. 58, 2713 (1985).

[12] L. Pavesi, J. Phys., Condens. Matter 15, 1169 (2003).

[13] Ant Ural, P.B. Griffin, J.D. Plummer, Appl. Phys. Lett. 79, 4328 (2001).

[14] Ryan Givens, O.F. Alcantara Bonfim, Am. J. Phys. 71, 87 (2003).

[15] P.S. Kireev, Physics of Semiconductors, High School, Moscow 1975, p. 584 (in Russian).

[16] J.L. Hastings, S.K. Estreicher, P.A. Fedders, Phys. Rev. B 56, 10215 (1997).

[17] V.V. Lukjanica, Phys. Techn. Semicond. 37, 422 (2003) (in Russian).

[18] J. Kawai, K. Hayashi, K. Okuda, A. Nisawa, The Rigaku Journal 15, 33 (1998).

[19] J.A. Van Vechten, Phys. Rev. B 10, 1482 (1974).

[20] R. Purlys, Ž. Norgela, B. Gricienè, J. Zaveckienè, Sci. Helth 2, 64 (2002) (in Lithuanian).

[21] A.J. Janavičius, Ž. Norgèla, R. Purlys, Acta Phys. Pol. A 104, 459 (2003).

[22] J.A. Van Vechten, Phys. Rev. B 38, 9913 (1988).

[23] B. Campbell, W. Choudhury, A. Mainwood, M. Newton, G. Davies, Nucl. Instrum. Methods Phys. Res. A 476, 680 (2002).

[24] R. Karazija, The Theory of X-ray and Electronic Spectra of Free Atoms. An Introduction, Mokslas, Vilnius 1987, p. 274.

[25] A.J. Janavičius, Ž. Norgèla, R. Purlys, Eur. Phys. J. Appl. Phys. 29, 127 (2005).

[26] P.T. Landsberg, Recombination in Semiconductors, Cambridge University Press, Cambridge 1991.

[27] H. Zillgen, P. Ehrhart, Mater. Res. Soc. Proc. 469, 175 (1997).

[28] V.V. Emtsev, T.V. Mashovets, V.V. Mikhnovich, Sov. Phys. Semicond. 26, 12 (1992).

[29] P.K. Kashkarov, International Soros Science Education Program 1, 105 (1999). 